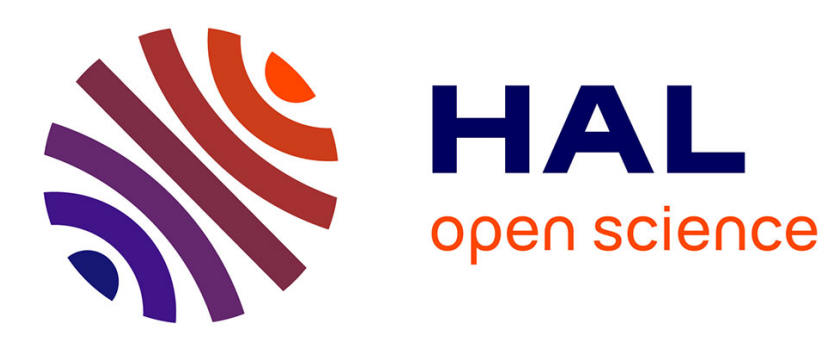

\title{
Experimental study of a hybrid electro-acoustic nonlinear membrane absorber
}

P. Y. Bryk, S Bellizzi, Renaud Côte

\section{To cite this version:}

P. Y. Bryk, S Bellizzi, Renaud Côte. Experimental study of a hybrid electro-acoustic nonlinear membrane absorber. Journal of Sound and Vibration, 2018, 424, pp.224-237. 10.1016/j.jsv.2018.03.014 . hal-01754878

\section{HAL Id: hal-01754878 \\ https://hal.science/hal-01754878}

Submitted on 30 Mar 2018

HAL is a multi-disciplinary open access archive for the deposit and dissemination of scientific research documents, whether they are published or not. The documents may come from teaching and research institutions in France or abroad, or from public or private research centers.
L'archive ouverte pluridisciplinaire HAL, est destinée au dépôt et à la diffusion de documents scientifiques de niveau recherche, publiés ou non, émanant des établissements d'enseignement et de recherche français ou étrangers, des laboratoires publics ou privés. 


\title{
Experimental study of a hybrid electro-acoustic nonlinear membrane absorber
}

\author{
P.Y Bryk ${ }^{a}$, S. Bellizzi ${ }^{a}$, R. Côte ${ }^{a}$, \\ ${ }^{a}$ Aix Marseille Univ, CNRS, Centrale Marseille, LMA \\ 4 impasse Nikola Tesla 13453 Marseille Cedex 13, FRANCE
}

\begin{abstract}
A hybrid electro-acoustic nonlinear membrane absorber working as a nonlinear energy sink (here after named EA-NES) is described. The device is composed of a thin circular visco-elastic membrane working as an essentially cubic oscillator. One face of the membrane is coupled to the acoustic field to be reduced and the other face is enclosed. The enclosure includes a loudspeaker for the control of the acoustic pressure felt by the rear face of the membrane through proportional feedback control. An experimental set-up has been developed where the EA-NES is weakly coupled to a linear acoustic system. The linear acoustic system is an open-ended tube, coupled on one side to the EA-NES by a box, and on the other side to a source loudspeaker by another box. Only sinusoidal forcing is considered. It is shown that the EA-NES is able to perform resonance capture with the acoustic field, resulting in noise reduction by targeted energy transfer, and to operate in a large frequency band, tuning itself passively to any linear system. We demonstrate the ability of the feedback gain defining the active loop to modify the resonance frequency of the EA-NES, which is a key factor to tune the triggering threshold of energy pumping. The novelty of this work is to use active control combined to passive nonlinear transfer energy to improve it. In this paper, only experimental results are analyzed.
\end{abstract}

Keywords: Noise Reduction, Hybrid Absorber, Nonlinear Energy Sink, Electroacoustic Absorber, High Sound Level, Low Frequency

Email address: cote@lma.cnrs-mrs.fr (R. Côte) 


\section{Introduction}

The reduction of noise and vibration at low frequencies is still nowadays a main issue in many fields of engineering. In order to overpass this issue, a new concept of absorbers including nonlinear behavior has been developed in

5 geted Energy Transfer" (TET) also named "energy pumping" [1. TET is an irreversible transfer of the vibrational energy from an input subsystem to a nonlinear attachment (the absorber) called Nonlinear Energy Sink (NES). TET permits to reduce undesirable large vibration amplitudes of structures or acousor through spatial energy localization by formation of nonlinear normal modes. The phenomena can be described as a 1:1 resonance capture 2 and, considering harmonic forcing, as response regimes characterized in terms of periodic and Strongly Modulated Responses (SMR) [3]. spring and a viscous linear damper. In the field of structural vibration, a wide variety of NES designs with different types of stiffness (cubic, non-polynomial, non-smooth nonlinearities...) has been proposed [4, 5, 6, 7]. In the acoustic field, to the best of our knowledge only one type of vibro-acoustic NES design has been tested, see the series of papers $[8,9,10,11,12$. It was demonstrated that a passive control of sound at low frequency can be achieved using a vibroacoustic coupling between the acoustic field (the primary system) and a geometrically nonlinear thin clamped structure (the NES). In [8, 9, 12, the thin baffled structure consists of a simple thin circular latex (visco-elastic) membrane whereas in

10 a loudspeaker used as a suspended piston is considered. In both cases, the thin baffled structure has to be part of the frontier of the closed acoustic domain (to be controlled). Hence only one face (named the front face) is exposed to the acoustic field whereas the other face (the rear face) radiates outside. Hence this type of devices has to be modified to be used in cavity noise reduction. 
A simple way to do this is to enclose the rear face of the thin clamped structure limiting the sound radiation. This principle has been used to design electroacoustic absorbers based on the use of an enclosed loudspeaker including an electric load that shunts the loudspeaker electrical terminals[13]. An electroacoustic absorber can either be passive or active in terms of their noise 35 suppression characteristics including as in [14, 15] pressure and/or velocity feedback techniques. Loudspeakers have also been used to design devices to control normal surface impedance [16. Two approaches have been developed. The first is referred to as direct control: the acoustic pressure is measured close to the diaphragm of the loudspeaker and used to produce the desired impedance. In the second approach, passive and active means are combined: the rear face of a porous layer is actively controlled so as to make the front face normal impedance take a prescribed value.

In this paper, a hybrid passive/active nonlinear absorber is developed. The absorber is composed of a clamped thin circular visco-elastic membrane with 45 its rear face enclosed. The acoustic field inside the hood (i.e the acoustic load of the rear face) is controlled using a loudspeaker with proportional feedback control. Three objective are assigned. Firstly, the device has to be designed such that it can be used inside a cavity. Secondly, noise reduction must mainly result from TET due to the nonlinear behavior of the membrane, thus defining 50 a new concept of NES. Thirdly, the control loudspeaker has to be used as a linear electroacoustic absorber inside the hood. The control loudspeaker does not act directly on the acoustic field to be reduced. It only modifies the relative acoustic load exciting the membrane. This absorber is here after named hybrid electroacoustic NES (EA-NES).

The paper is organized as follows. In Section 2 , the principle of functioning of the EA-NES under study is described considering each sub-structure separately. In Section 3, we first describe the experimental set-up. It is composed of an acoustic field (in a pipe, excited by a loudspeaker) coupled to the EA-NES. Then we check the stability analysis of the feedback loop and perform a frequency analysis under broadband excitation. In Section 4, we analyze in detail the 
responses under sinusoidal excitations and we bring some confirmations on the efficiency of the EA-NES.

\section{The Hybrid Electro-Acoustic NES}

\subsection{General presentation}

The EA-NES is shown in Fig. 1. It is composed of a clamped circular latex membrane with one face (the front face) exposed to the acoustic field to be reduced and the other one (the rear face) enclosed. The hood includes a feedback loop composed of a microphone, a loudspeaker and a unit control. The feedback loop controls the acoustic pressure inside the hood and seen by the rear face of the membrane.

(a)

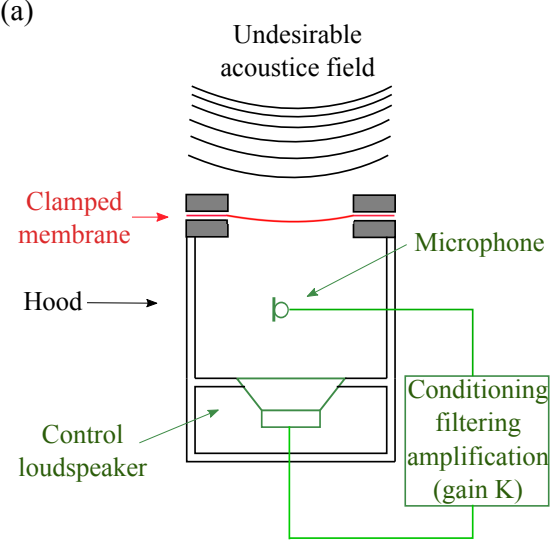

(b)

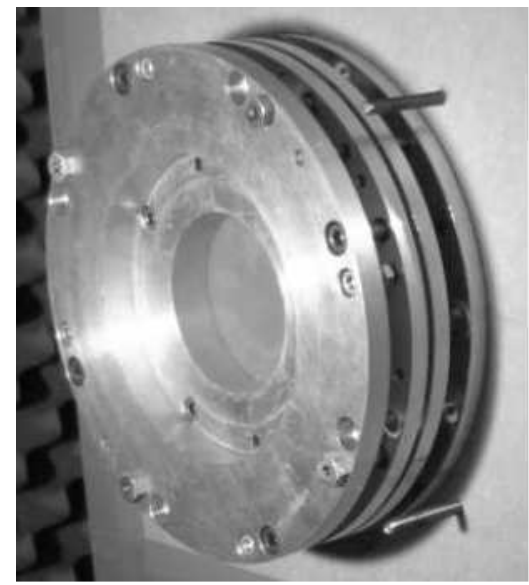

Figure 1: EA-NES: (a) Schematic representation and (b) Front face.

The EA-NES is based on the conjugate functioning of three elements :

- The clamped membrane that interacts with the acoustic field in order to provide noise attenuation in its non-linear range;

75

- The hood by which the EA-NES can work inside a surrounding acoustic field unlike previous developed NES (see for example [9]); 
- The feedback loop that reduces the pressure in the hood allows to use a small hood volume and also to tune the stiffness and damping linear behaviour of the EA-NES.

\subsubsection{The clamped membrane}

The clamped membrane with its supporting device is shown in Fig. 1(right). It was already used in $[9]$. The device allows to change the diameter of the membrane (from 40 to $80 \mathrm{~mm}$ ). It includes a sliding system used to apply a stress is set, the membrane is clamped to the supporting device. Applying an in-plane pre-stress modifies the modal component of the associated underlying linear system.

Coupled to an undesirable acoustic field, the clamped membrane device Direct [12] or indirect [9] couplings are possible. If the clamped membrane device is properly designed, TET is obtained thanks to resonance capture phenomena. Resonance capture occurs between two nonlinear modes resulting of a weak coupling between the nonlinear subsystem (the NES) and the linear subsystem respectively with the linear mode of the NES and the target mode of the acoustic field. Hence, when the in-plane pre-stress of the clamped membrane sets the resonance frequency of the NES lower than the resonance frequency of the target mode of the acoustic field, TET is possible above a threshold excitation level. Furthermore, Bellet 17] has also shown that the gap between the two resonance frequencies has an impact on the threshold of the TET: the closer are the two resonance frequencies, the smaller is the threshold of the TET.

As a subsystem of the EA-NES, the clamped membrane provides the coupling between the EA-NES and the acoustic field to reduce. It is also responsible for the resonance capture phenomena (as a nonlinear component). The other subsystems (the hood and the feedback loop) must preserve the essential prop- 
erties of a NES, namely a weakly damped system with a hardening nonlinear mode, and with a frequency at low vibratory amplitude smaller than the resonance frequency of the target mode of the acoustic field.

\subsubsection{The hood}

The hood is a wooden cubic box with the clamped membrane fixed on one face and an enclosed control loudspeaker on the opposite face (see Fig. 1(left)). The hood has been added to meet two key objectives. Firstly, with hood, the EA-NES can be used to reduce the noise in a cavity where only the front face of the clamped membrane is load by the undesirable acoustic field. Secondly, the hood creates a difference of pressure between the two faces of the membrane, which permits to place it anywhere inside the acoustic field.

When the acoustic wavelength is large in comparison with the largest dimension of the hood, the acoustic field can be considered as homogeneous in the hood and the acoustic pressure $p_{e}(t)$ can be related to the relative variation of the volume as

$$
p_{\mathrm{e}}(t)=-\rho_{\mathrm{a}} c_{0}^{2} \frac{\Delta V_{\mathrm{e}}(t)}{V_{\mathrm{e}}}
$$

where $V_{\mathrm{e}}$ denotes the volume of the box at rest, $\Delta V_{\mathrm{e}}(t)$ the variation of the volume, $\rho_{\mathrm{a}}$ the volumetric mass of the air and $c_{0}$ the celerity of sound in the air. Assuming the motion of the membrane primarily defined on the first transversal mode, Eq. (1) reduces to

$$
p_{\mathrm{e}}(t)=\frac{\rho_{\mathrm{a}} c_{0}^{2}}{V_{\mathrm{e}}}\left(S_{\mathrm{m}}^{\mathrm{e}} x_{\mathrm{m}}(t)-S_{\mathrm{LS}}^{\mathrm{e}} x_{\mathrm{LS}}(t)\right)
$$

where $x_{\mathrm{m}}$ denotes the transversal membrane motion with $S_{\mathrm{m}}^{\mathrm{e}}$ the effective area of the membrane and $x_{\mathrm{LS}}$ denotes the transversal motion of the diaphragm of the loudspeaker with $S_{\mathrm{LS}}^{\mathrm{e}}$ the effective area.

Eq. (2) means that the air inside the hood is equivalent to an acoustic compliance that resists to the motion of the membrane and hardens it. This compliance being in return inversely related to the volume $V_{e}$, it results that the smaller is the volume of hood, the higher is the resonance frequency of the underlying 
linear EA-NES. Finally Eq. (2) also shows that the acoustic pressure $p_{\mathrm{e}}(t)$ can be reduced to zero if $x_{\mathrm{LS}}$ and $x_{\mathrm{m}}$ are in-phase.

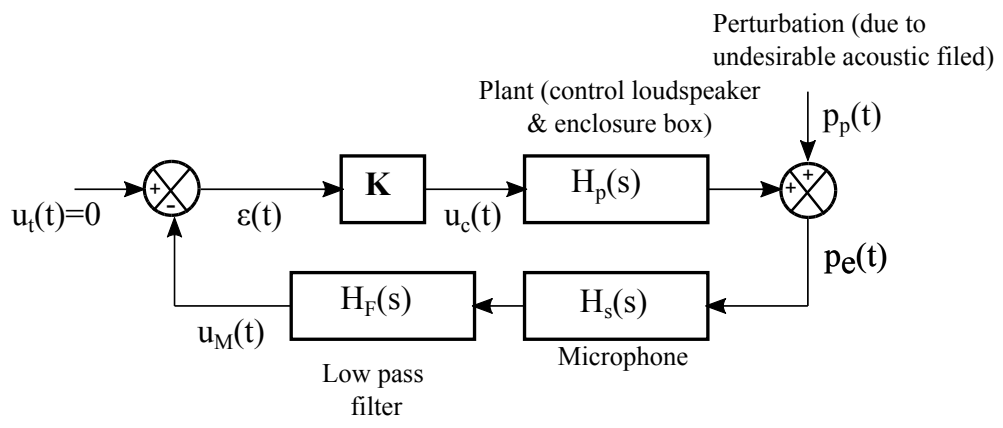

Figure 2: Block diagram of the proportional controller.

The feedback loop is composed of an enclosed loudspeaker inside the hood, a microphone placed at the geometrical center of the hood and a unit control (see Fig. 1(left)). The feedback loop is based on a proportional controller following the block-diagram displayed in Fig. 2 where $H_{\mathrm{p}}$ (the plant transfer) denotes the transfer function between the tension $u_{\mathrm{c}}(t)$ applied to the control loudspeaker (voltage-feedback control) and the acoustic pressure $p_{\mathrm{e}}(t)$ and $H_{\mathrm{s}}$ the transfer function characterizing the microphone. A part of the acoustic pressure $p_{\mathrm{e}}(t)$ is due to $p_{\mathrm{p}}(t)$ (perturbation term) resulting from the undesirable acoustic field acting on the front face of the clamped membrane. The unit control includes an analogue band-pass filter $H_{\mathrm{F}}$ and a controller sets a scalar (real) gain $K$.

The reference of the loop $\left(u_{\mathrm{t}}(t)=0\right)$ means a pressure reduction $p_{\mathrm{e}}(t)$ until zero, which is only possible with an infinite value of $K$ as illustrated by the following total loop transfer in Laplace variables

$$
P_{\mathrm{e}}(s)=\left(1-H_{\mathrm{p}}(s) K H_{\mathrm{F}}(s) H_{\mathrm{s}}(s)\right)^{-1} P_{\mathrm{p}}(s)
$$

between the acoustic pressure $p_{\mathrm{p}}(t)$ and the acoustic pressure $p_{\mathrm{e}}(t)$. However the choice of the gain $K$ is limited by instability phenomena which exist usually for positive gain $K$ at high frequencies, resulting from the acoustic modes of 
the cavity and for negative gain $K$ at low frequency $(<100 \mathrm{~Hz})$, resulting from the control loudspeaker and membrane dynamics. The influence of the acoustic modes of the cavity is reduced by placing the microphone at the geometrical center of the hood. This location is near the node of pressure of some of the first modes.

Stability margin analysis results from the properties of the Open Loop Transfer Function (OLTF) between $\epsilon(t)$ and $u_{\mathrm{M}}(t)$ defined by $H_{\mathrm{F}}(s) H_{\mathrm{S}}(s) H_{\mathrm{p}}(s) K$ (see Fig. (2)). The variation range of the gain values can be increased by selecting adequately the filter $H_{\mathrm{F}}(s)$. It is that we do in the next section.

\subsection{Choice and sizing of components of the EA-NES}

Our objective is to design a EA-NES able to interact with primary acoustic fields in the frequency range $[40,100] \mathrm{Hz}$.

The clamped latex membrane was designed following the recommendations discussed in 9 . The membrane has a radius of $0.05 \mathrm{~m}$ and a thickness of $0.0002 \mathrm{~m}$. These dimensions guarantee a proper functioning of the clamped latex membrane as a NES when the membrane is coupled to a resonant tube [9, 11] without hood (alone). The sliding system is adjusted such that the pre-stress of the membrane gives the coupled setup (EA-NES and primary system) a resonance associated to the EA-NES at a frequency around $70 \mathrm{~Hz}$ (see Fig. 5).

The dimensions of the enclosure are $0.38 \mathrm{~m} \times 0.22 \mathrm{~m} \times 0.22 \mathrm{~m}$, which gives $V_{\mathrm{e}}=$ $0.018 \mathrm{~m}^{3}$. The membrane and the control loudspeaker are fixed respectively on the opposite faces with size $0.22 \mathrm{~m} \times 0.22 \mathrm{~m}$. Note that the pressure $p_{\mathrm{e}}(t)$ inside the volume $V_{\mathrm{e}}$ is equivalent to a compliance as long as the acoustic wavelength

175 is large in comparison with the largest dimension of the enclosure. With $\lambda>$ $10 \times 0.38$ one obtains a maximum frequency of $f_{\max }=89.5 \mathrm{~Hz}$.

Efficient control loudspeakers need the following properties: high resonance frequency of the driver part (such that the resonance frequency of the enclosed loudspeaker is above the working frequency band of the EA-NES), large force factor, linear behavior for large excursion of the diaphragm and effective area of the diaphragm as large as possible compared to the effective area of the 
latex membrane. Note that the area of the diaphragm is also limited by the size of the boxes. The BEYMA 8P300Fe/N (8 Inch) loudspeaker family was selected corresponding to the following Thiele/Small parameter values: $m_{\mathrm{LS}}=$ $27 \times 10^{-3} \mathrm{~m}^{3}$. An Audax HT-240G0 loudspeaker is used as an acoustic source.

Two experiments are conducted, one using a tube of length $L_{1}=2.1 \mathrm{~m}$ as a primary system and the other with a tube of length $L_{2}=1.8 \mathrm{~m}$. The objective is 

systems having different resonance frequencies.

(a)

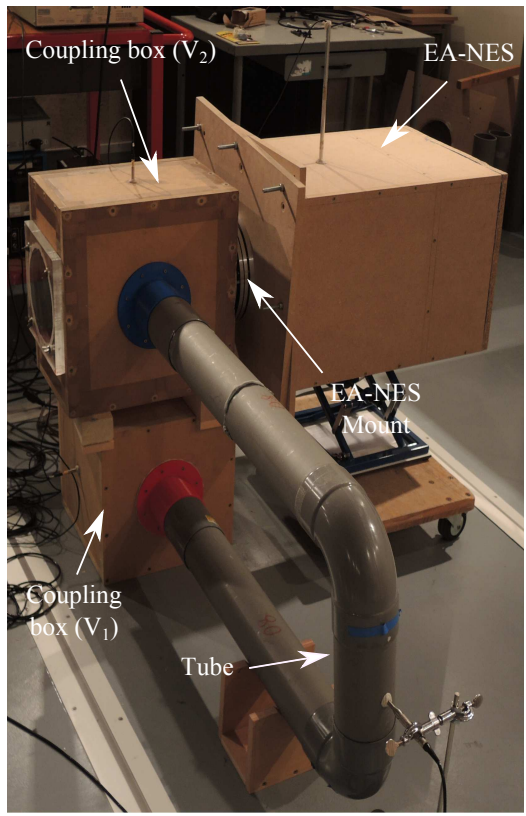

(b)

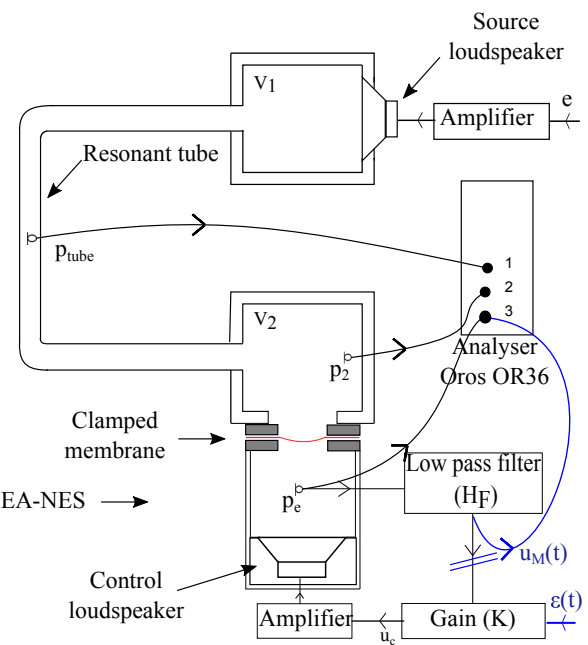

Figure 3: Experimental set-up under study: (a) Picture and (b) Schema (the blue lines define the configuration used to measure the OLTF and the same notations as in Fig. 2 were used).

During the measurement, a target voltage signal $e(t)$ from a generator (not shown in Fig. 3) and a power amplifier (TIRA, BAA120) provide an input current or input voltage signal to the source loudspeaker (depending the selected driving mode: current-or voltage-feedback control mode). The responses of the system are recorded simultaneously (using a multi-channel analyzer/recorder OROS, OR38): the acoustic pressures $p_{\text {tube }}(t)$ (at the mid-length of the tube), $p_{2}(t)$ (inside the box 2) and $p_{\mathrm{e}}(t)$ (inside the hood of the EA-NES) with three microphones (GRAS, 40BH), (see Fig. 3. right). Also recorded (not shown on the schema) are the source loudspeaker current $i_{\mathrm{s}}(t)$ and voltage $e_{\mathrm{s}}(t)$ responses and the control loudspeaker current $i_{\mathrm{c}}(t)$ and voltage responses $e_{\mathrm{c}}(t)$. The sampling frequency is $f_{\mathrm{s}}=8192 \mathrm{~Hz}$.

Figure 4 shows the Frequency Response Function (FRF) denoted $p_{2} / e$ between the voltage applied to the source loudspeaker $e(t)$ (in voltage-feedback 
(a)
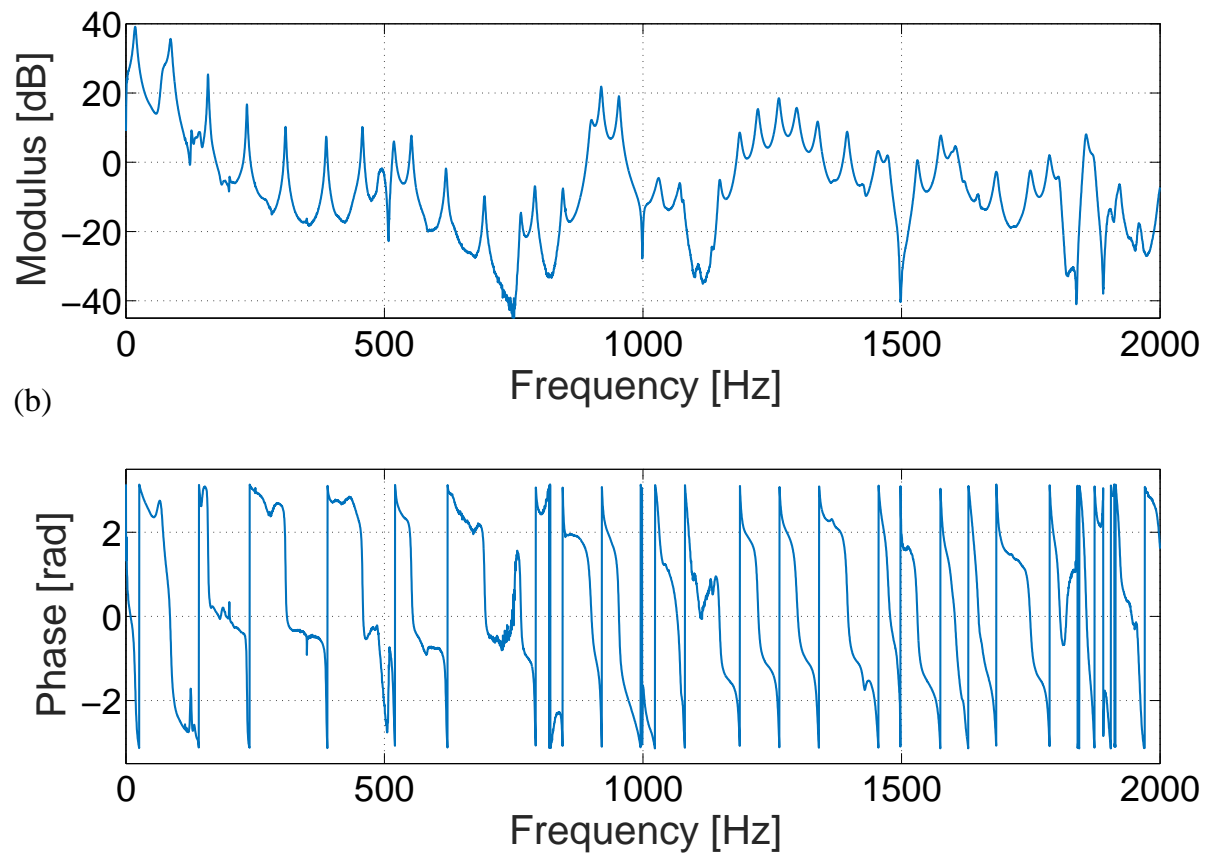

Figure 4: FRF $p_{2} / e$ measured with the EA-NES with $K=0$ coupled to the tube of $L_{1}$ length: (a) Modulus and (b) Phase.

control mode) and the acoustic pressures $p_{2}(t)$ with the tube of $L_{1}$ length and imposing the gain $K=0$ (passive configuration, corresponding to a short-circuit between the loudspeaker connectors). For convenience, we use notation in time domain $\left(p_{2}\right.$ and $e$ ) to characterize FRF. The source loudspeaker is voltage-driven so that its own resonance is damped and do not disturb the other resonances of the system. A band limited $([10,2000] \mathrm{Hz})$ white-noise is used and limited to a low excitation level.

The lowest resonance around $20 \mathrm{~Hz}$ results from the whole coupled system (the tube acts as an acoustic mass coupled to the coupling boxes acting like springs). The next two resonances, around $72 \mathrm{~Hz}$ and $87 \mathrm{~Hz}$, as seen in Fig. 5 . are respectively assigned to the EA-NES and to the first mode of the tube alone. Beyond $100 \mathrm{~Hz}$, the resonance frequencies coming from the highest modes of the 
tube and the coupling boxes appear. From now on we will focus on a frequency span below $100 \mathrm{~Hz}$.

Figure 5 shows the FRF $p_{\text {tube }} / e$ and $p_{2} / e$ measured with the tube of $L_{1}$ length and with the tube of $L_{2}$ length and imposing in both cases the control gain $K=0$. For the two tubes, two resonance peaks appear in the frequency band $[40,120] \mathrm{Hz}$. In both cases ( $L_{1}$ and $L_{2}$ lengths), the first resonance peak is localized around the frequency $70 \mathrm{~Hz}$ and can be attributed to the EA-NES. The second resonance peak (around the frequency $87 \mathrm{~Hz}$ for the tube of $L_{1}$ length and around the frequency $99 \mathrm{~Hz}$ for the tube of $L_{2}$ length) is primarily attributed to the tube (in accordance with $L_{1}<L_{2}$ ). In both case, they exhibit high response levels.
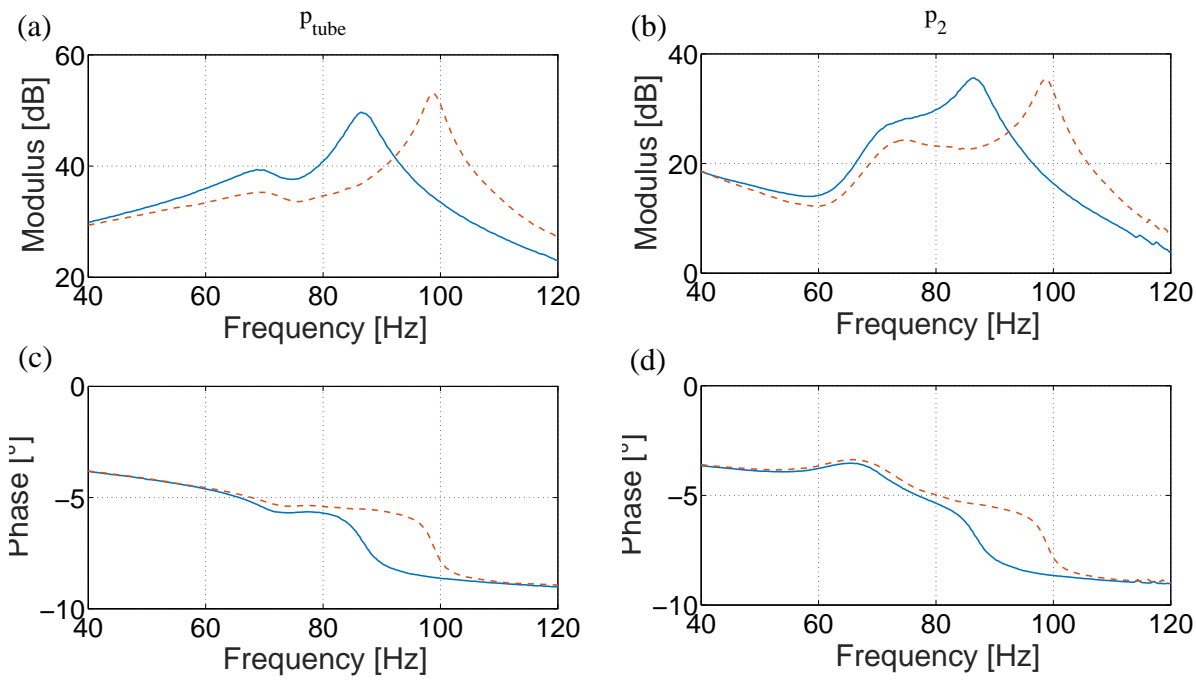

Figure 5: FRFs $(\mathrm{a}, \mathrm{c}) p_{\text {tube }} / e$ and (b,e) $p_{2} / e$ measured with the EA-NES with $K=0$ coupled to the tube of $L_{1}$ (blue curves) and $L_{2}$ (red dashed curves) length: (a,b) Modulus and (c,d) Phase.

\subsection{Stability of the feedback loop}

As a preliminary step, the OLTF, $H_{\mathrm{F}}(s) H_{\mathrm{S}}(s) H_{\mathrm{p}}(s) K$ at $s=\mathrm{j} 2 \pi f$, is mea250 sured with the EA-NES coupled to the tube of length $L_{1}$ with a unity gain $(K=1)$ (see Fig. 3 (blue indications)). The acoustic source is off. The input 
signal $\epsilon(t)$ is a band limited $([10,4000] \mathrm{Hz})$ white-noise. We record the Frequency Response Function $u_{\mathrm{M}} / \epsilon$. The set-up is not shown. The KEMO band-filter is set to a 4 Pole Butterworth low-pass filter with cut-off frequency $2048 \mathrm{~Hz}$ and $0 \mathrm{~dB}$ gain. With this setting, the gain margin $G_{\mathrm{m}}$ increases up to $52 \mathrm{~dB}$. The corresponding OLTF is shown in Fig. 6 in the Nyquist domain. Also reported is the OLTF measured with the tube of $L_{2}$ length. The two curves are similar and define close gain margins (equal to $52.7 \mathrm{~dB}$ with the tube of $L_{2}$ length).

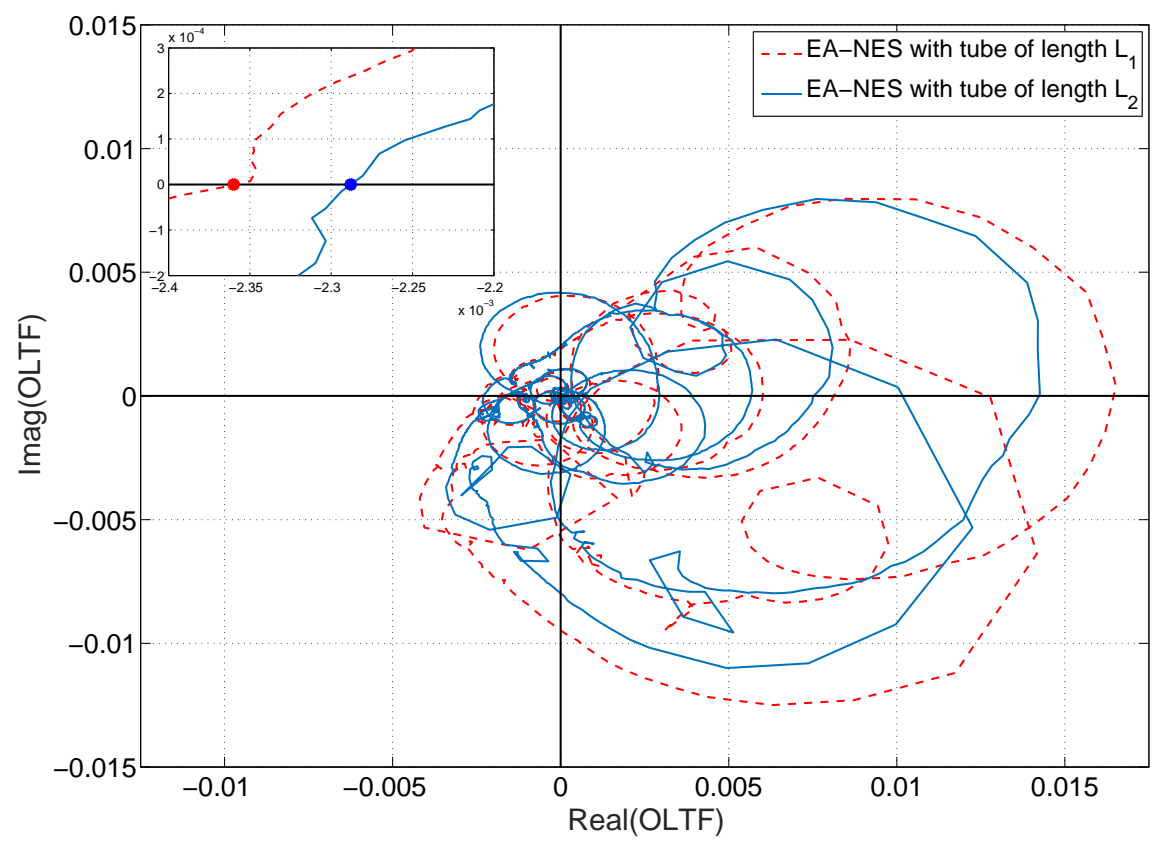

Figure 6: Open loop transfer function in the Nysquist domain measured with the EA-NES with unity gain $(K=0)$ coupled to the tube of $L_{1}$ (red dashed curves) and $L_{2}$ (blue curves) length.

Selecting the gain $K$ in the range $[0,200]$ results in an OLTF with gain margin greater than or equal to $6 \mathrm{~dB}$, the $0 \mathrm{~dB}$ gain margin corresponding to $K=400$. Gain margins and phase margins with the associated critical frequencies are reported Table 1 for several values of $K$. Similarly selecting the gain $K$ in the range $[-64,0]$ results in an OLTF with gain margin from to $0 \mathrm{~dB}$ 
to $36 \mathrm{~dB}$.

\begin{tabular}{|c|c|c|c|c|c|c|c|c|}
\hline$K$ & \multicolumn{2}{|c|}{$f_{\mathrm{G}_{\mathrm{m}}}(\mathrm{Hz})$} & \multicolumn{2}{c|}{$G_{\mathrm{m}}(\mathrm{dB})$} & \multicolumn{2}{c|}{$f_{\mathrm{P}_{\mathrm{m}}}(\mathrm{Hz})$} & \multicolumn{2}{|c|}{$P_{\mathrm{m}}\left(^{\circ}\right)$} \\
\hline & $L_{1}$ & $L_{2}$ & $L_{1}$ & $L_{2}$ & $L_{1}$ & $L_{2}$ & $L_{1}$ & $L_{2}$ \\
\hline-1 & 75 & 76.25 & 36.1 & 36.9 & - & - & 360 & 360 \\
\hline 1 & 441 & 436 & 52. & 52.7 & - & - & 360 & 360 \\
\hline 50 & 441 & 436 & 18. & 18.7 & - & - & 360 & 360 \\
\hline 100 & 441 & 436 & 12. & 12.7 & 131.9 & 133 & 93 & 102 \\
\hline 200 & 441 & 436 & 6. & 6.7 & 207 & 203 & 45 & 40 \\
\hline
\end{tabular}

Table 1: Gain $G_{\mathrm{m}}$ and phase $P_{\mathrm{m}}$ margins and associated frequencies $f_{\mathrm{G}_{\mathrm{m}}}$ and $f_{\mathrm{P}_{\mathrm{m}}}$ of the feedback loop measured with the EA-NES for several values of the gain $K$ coupled to the tubes of $L_{1}$ and $L_{2}$ length.

. Note that, one can also modify the use of the feedback loop in order to amplify $p_{\mathrm{e}}(t)$ instead of reducing it (hardening the clamped membrane behaviour). To achieve this goal, the loudspeaker is fed with a negative value for the gain $K$. This case is equivalent to study the feedback loop with OLTF shifted of 180 degrees, which gives different phase and gain margins reported in Table 1 for $K=-1$. One can notice that the gain margin for $K=1$ is higher than the gain margin with $K=-1$. It means the feedback loop can soften more the membrane than harden it.

\subsection{The gain $K$ as a tuning parameter of the EA-NES}

The objective of this section is to verify that the gain $K$ in the feedback loop can be used to tune the modal component associated with the EA-NES without disturbing the modal component associated with the primary system. The influence of the gain $K$ on the behavior of the EA-NES is analyzed after measuring the FRF $p_{2} / e$. Here also the source loudspeaker is driven in voltagefeedback control mode. It is excited with a low-level band-limited white-noise.

Figure 7 shows the modulus of the FRF $p_{2} / e$ with the tube of $L_{1}$ length for several values of the gain $K$. Also reported is the FRF obtained by replacing the EA-NES with the clamped membrane NES alone (no hood). First of all, we 


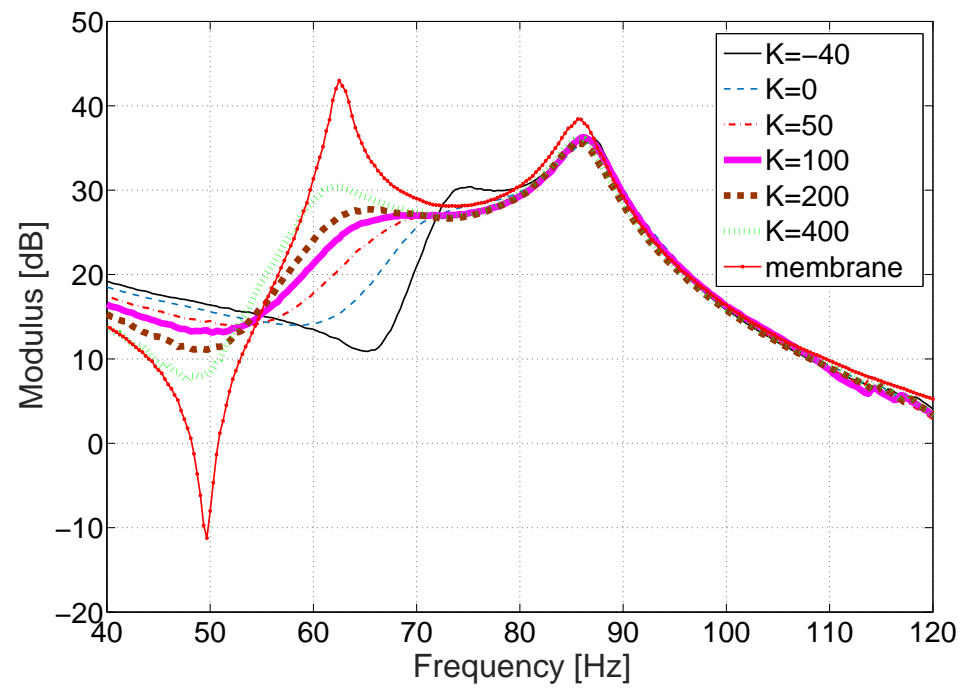

Figure 7: FRF $p_{2} / e$ measured with the EA-NES for several values of the gain $K$ and with a clamped membrane NES (red continuous line) coupled to the tube of $L_{1}$ length.

observe that the resonance peak around $f=87 \mathrm{~Hz}$ and the associated bandwidth is not affected by the change in the value of $K$. In contrast, starting from as expected, negative values of the gain $K$ increase the resonance frequency. 


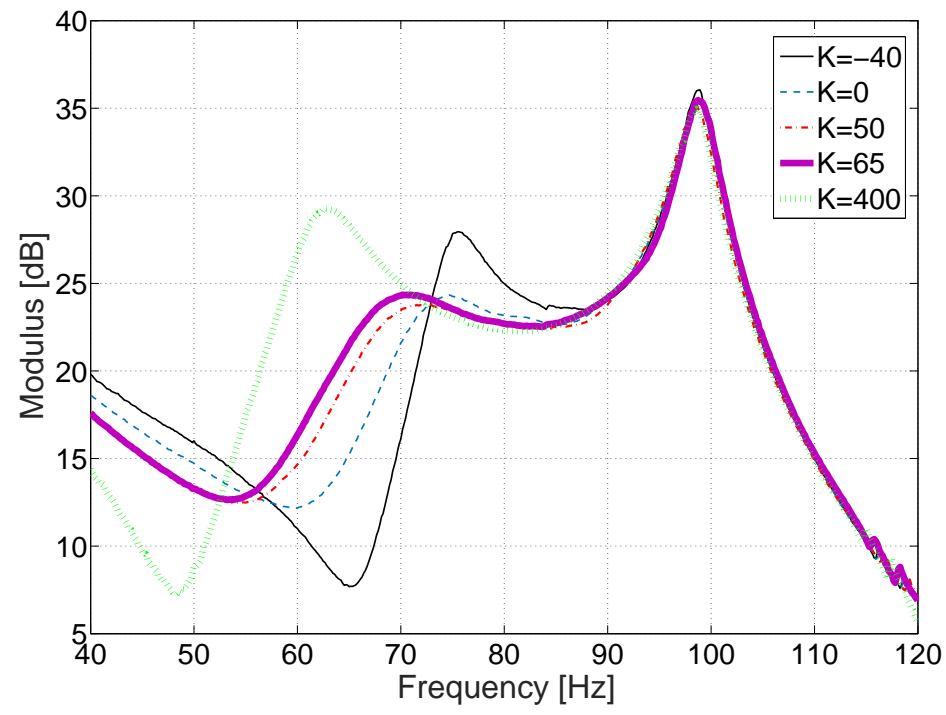

Figure 8: FRF $p_{2} / e$ measured with the EA-NES for several values of the gain $K$ coupled to the tube of $L_{2}$ length.

Similar behaviors are obtained with the same EA-NES mounted on the tube of $L_{2}$ length. The modulus of the FRF $p_{2} / e$ are reported in Fig. 8. The important fact is that the last observation has always been possible, with the two lengths and of course for any intermediate length. The feedback loop of the EANES is able to work as a linear electroacoustic absorber inside the hood with any linear primary system having its resonance frequency in a large frequency range.

\section{Study at high excitation level: efficiency of the EA-NES from TET}

Now let us look at the behavior of the coupled system under a sinusoidal forcing when the frequency and the amplitude of the sinusoidal forcing vary. Here the source loudspeaker is driven using a current-feedback control mode reducing the dissipation introduced in the system by the source loudspeaker 18 .

During the measurement, a target signal $e(t)$ from a generator (TTI TGA1244) (not shown in Fig. 3) and a power amplifier (TIRA, BAA120) provide an input 
current signal to the source loudspeaker. The target signal $e(t)$ is of the form

$$
e(t)=E \cos \left(2 \pi f_{\mathrm{e}} t+\phi_{\mathrm{e}}\right)
$$

where $f_{\mathrm{e}}$ denotes the excitation frequency and $E$ is the associated excitation amplitude. The phase $\phi_{\mathrm{e}}$ is introduced arbitrarily by the signal generator.

A measurement run consists in making a series of experiments, where the value of the scanning frequency $f_{\mathrm{e}}$ is updated for each experiment, while the other parameter $E$ remains unchanged. The duration of an experiment must be limited for practical reasons, but must be long enough to capture the physics of the response. We have chosen a duration of $13 \mathrm{~s}$ for each experiment. There are two steps in an experiment. The first step lasts $3 \mathrm{~s}$ with no source signal. It permits us to get null initial conditions, whatever happened before. The second step lasts $10 \mathrm{~s}$ with the source on, but we record only the last $7 \mathrm{~s}$, the first $3 \mathrm{~s}$ include generally the transient effects of the excitation.

A measurement test consists in making a series of runs where the value of the amplitude $E$ is updated for each run. Six tests were performed with the tube of $L_{1}$ length with the following six values of the gain $K:-40,0,50,100$, 200 and 400. Five tests were performed with the tube of $L_{2}$ length with the following five values of the gain $K:-40,0,50,65$ and 100 . The frequency step $\delta f=0.25 \mathrm{~Hz}$ was used to define runs in the frequency band $[80,93] \mathrm{Hz}$ for the tube of $L_{1}$ length and $[90,105] \mathrm{Hz}$ for the tube of $L_{2}$ length. The step in the amplitude band [0.01414, $E_{\max }$ ] is equal to 0.07 with $E_{\max }=2.9$ for the tube of $L_{1}$ length and $E_{\max }=4$ for the tube of $L_{2}$ length.

In order to characterize the pumping phenomenon, we first describe in details the results obtained with the EA-NES with $K=200$ coupled to the tube of $L_{1}$ length, followed by an analysis of the influence of $K$. Then, the experiment involving the tube of $L_{2}$ length is considered.

\subsection{EA-NES with the tube of $L_{1}$ length and $K=200$}

The behavior of the system with the tube of $L_{1}$ length and $K=200$ under sinusoidal excitation, scanned around the first mode of the tube is presented. 


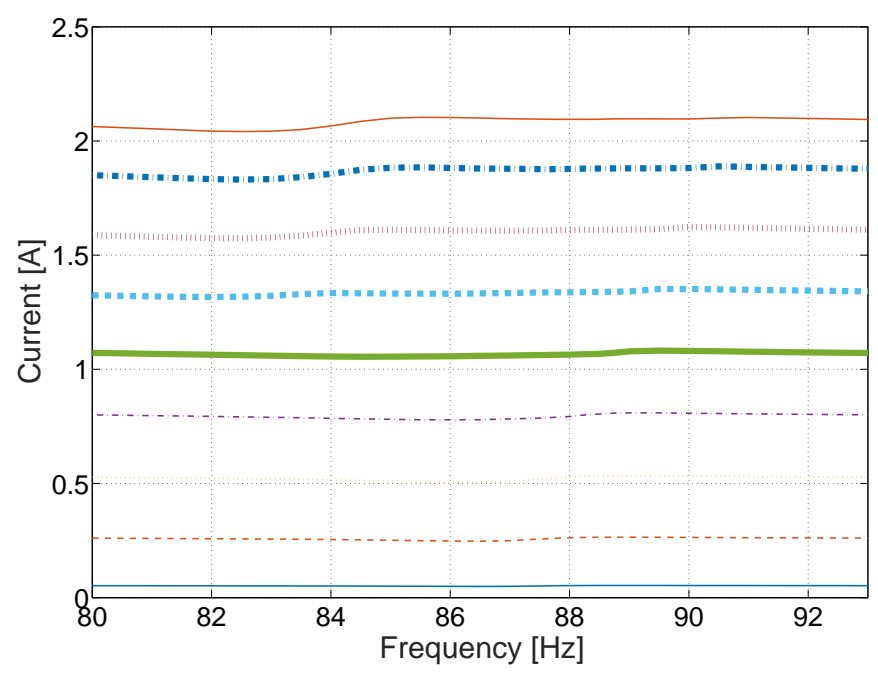

Figure 9: RMS values of the source loudspeaker current $i_{\mathrm{S}}(t)$ measured with the EA-NES with $K=200$ coupled to the tube of $L_{1}$ length for several values of the excitation amplitude $E$ versus excitation frequency.

Concerning the command level of the source loudspeaker, the power amplifier driving the loudspeaker in current-feedback mode provides a nearly constant source loudspeaker current $i_{\mathrm{s}}(t)$ as shown in Fig. 9 where the Root Mean Square (RMS) values of $i_{\mathrm{s}}(t)$ are plotted versus frequency for some excitation amplitudes. Hence the source loudspeaker is not modified by the system (tube+EANES) and it plays its full role as a controlled source.

Concerning the system response, the RMS value in the steady state regime is extracted from each measurement of the acoustic pressure $p_{\text {tube }}(t)$ and it is plotted in Fig. 10(a) as a surface level depending on the excitation frequency and the RMS value of the excitation amplitude. As expected a resonance peak around $f=87 \mathrm{~Hz}$ is observed on the sound pressure response at low excitation level. The flat surface a low excitation level becomes, due to the nonlinear behaviour of the clamped membrane, substantially disturbed by an increase in the excitation level. Abrupt changes appear accompanied by a reduction of the response level and the occurrence, at high excitation level, of a resonance 
(a)

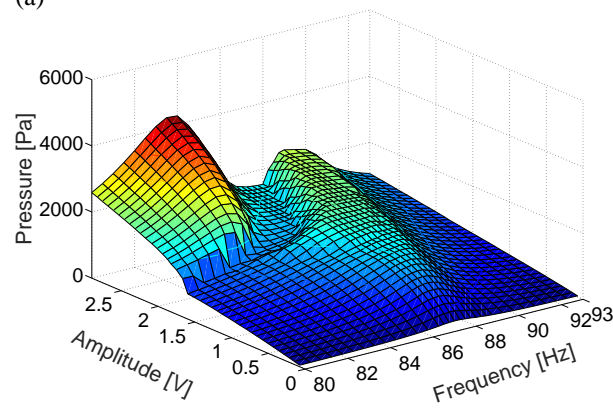

(c)

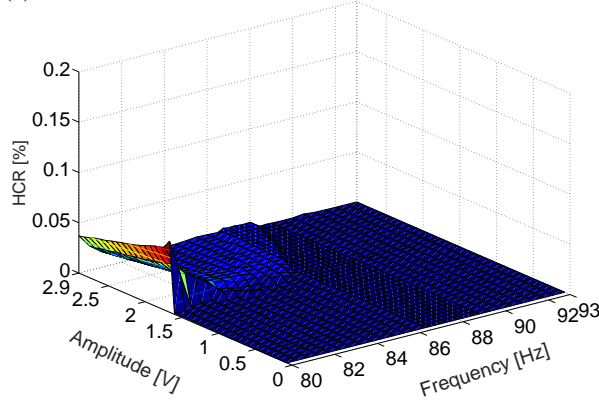

(b)

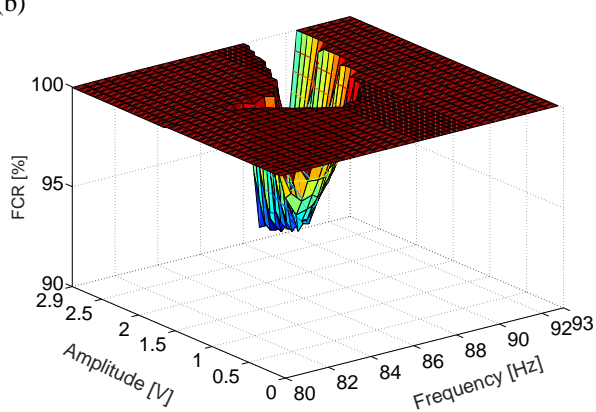

(d)

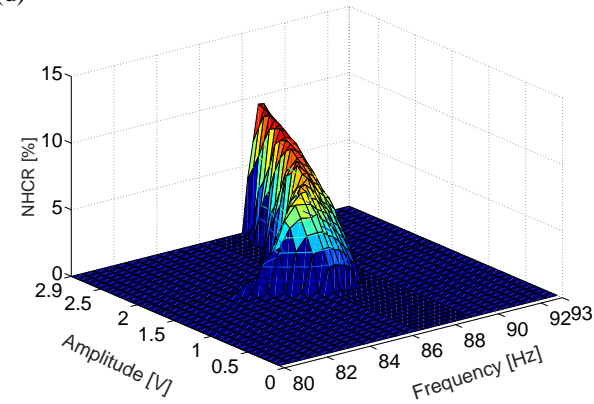

Figure 10: System with the EA-NES with $K=200$ coupled to the tube of $L_{1}$ length: (a) RMS values, (b) FCR, (c) HCR and (d) NHCR of the steady state regime of $p_{\text {tube }}$ as a surface level according to frequency and excitation amplitude.

peak around $f=83.5 \mathrm{~Hz}$, smaller than the resonance frequency observed at low excitation level. These behaviors were classically reported considering NES analysis 9 and they can be attributed to the pumping phenomenon and TET from the primary system to the NES. TET signatures are of two types. First, TET occurs only when the primary linear system reaches a certain vibration energy threshold and secondly TET is associated to quasi-periodic response regime with a slow evolution of the amplitudes (also named Strongly Modulated Regimes (SMR) [3]). This point can be confirmed by an analysis of energy conversion.

To analyze the energy conversion occurring from the fundamental frequency $f_{\mathrm{e}}$, to the harmonic frequencies $\left(k f_{\mathrm{e}}\right.$ for the integer $\left.k>1\right)$ and to the non harmonic frequencies $\left(f \neq k f_{\mathrm{e}}\right)$, the Fundamental Conversion Ratio (FCR), the 
Harmonic Conversion Ratio (HCR) and the Non Harmonic Conversion Ratio (NHCR) are used. The definition are these indicators are recalled in[11]. For each signal, FCR, HCR and NHCR are obtained from Fourier analysis estimated from the steady state response. Basically, the FCR is the proportion of signal energy at the source frequency. For a linear system this indicator should be $100 \%$. The HCR is the proportion of signal at the integer harmonics of the source frequency. It can give information about nonlinear effects like saturation. The NHCR is the proportion of signal frequencies out of the integer harmonics 375 of the source frequency. It can give information about nonlinear effects like a loss of periodicity.

FCR (respectively HCR and NHCR) are plotted in Fig. 10(b) (respectively (c) and (d)) as a surface level depending on the excitation frequency and the excitation amplitude. At low excitation amplitude $(E \leq 1)$, the energy of the steady state responses are concentrated on the fundamental frequency (see Fig. 10(b)). The corresponding response regimes are periodic resulting from the linear behavior of the EA-NES. By increasing the excitation amplitude, an amplitude-frequency domain appears, characterized by steady state responses, a fraction of energy of which is transferred from the fundamental frequency to the non harmonic frequency domain (see Fig. 10(d)). This transfer of energy increases with the excitation amplitude but the associated RMS level of the acoustic pressure in the tube remains limited (see Fig. 10(a)). In this domain, the responses of the system are no more periodic and are replaced by so-called SMR. This domain is the domain where the EA-NES works well.

Another amplitude-frequency domain which is detached from the previous domain is also visible in Fig. 10(c) at low frequencies and high amplitudes showing that, in this case, energy can be transferred from the fundamental frequency to the high harmonic frequencies. In this domain, the response of the system is periodic but unfortunately the associated RMS level increases, which leads to undesired periodic regimes. This domain is the domain where is EANES not efficient for noise reduction and it is characterized by the appearance of undesired periodic regimes. 


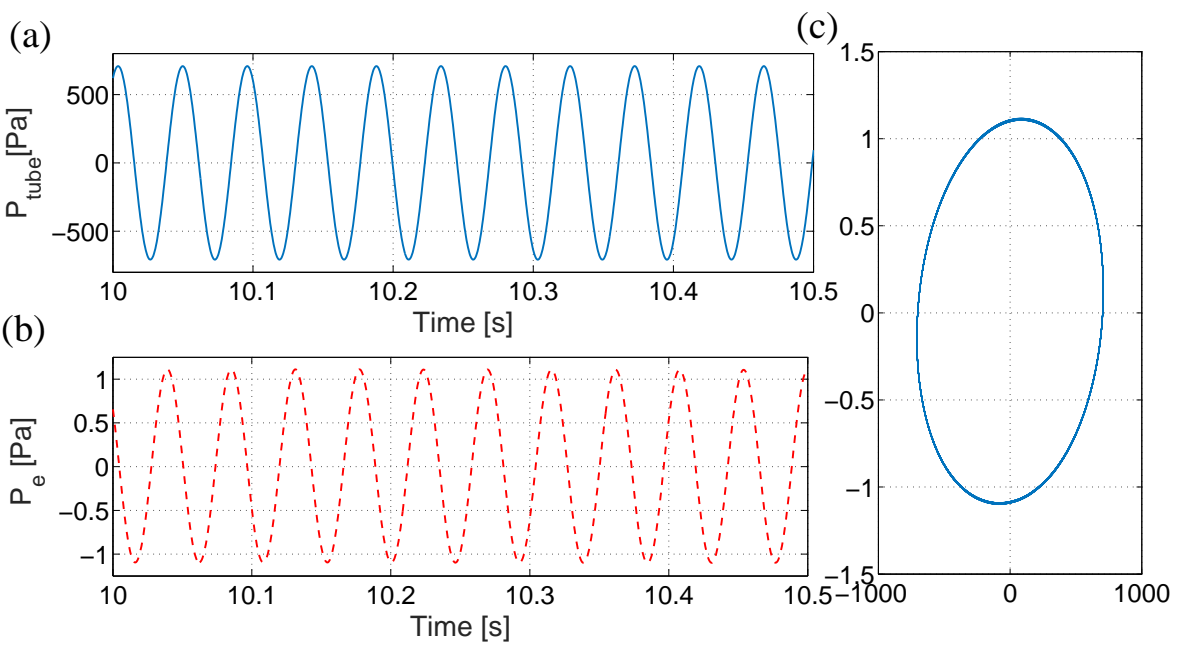

Figure 11: System with the EA-NES with $K=200$ coupled to the tube of $L_{1}$ length: Steady state responses of (a) $p_{\text {tube }}$ and (b) $p_{\mathrm{e}}$ versus time and (c) parametric plot ( $p_{\text {tube }}, p_{\mathrm{e}}$ ) obtained with the excitation frequency $f_{\mathrm{e}}=86.75$ and excitation amplitude $E=0.29$.

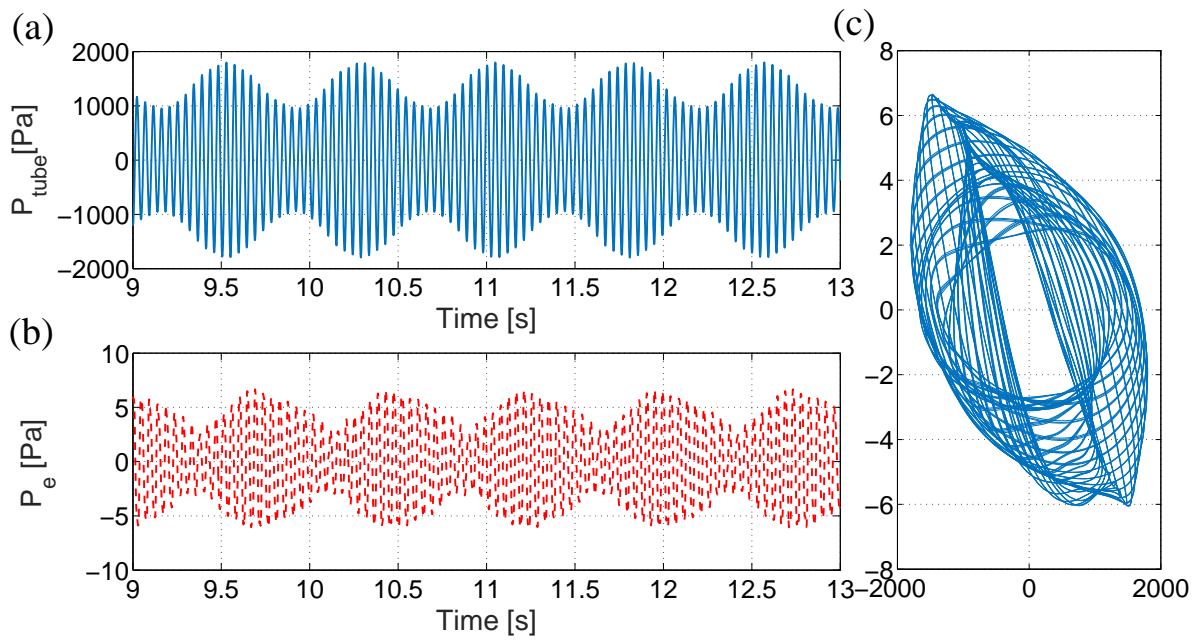

Figure 12: Idem as Fig. 11 with the excitation frequency $f_{\mathrm{e}}=86.75$ and excitation amplitude $E=1.5$. 


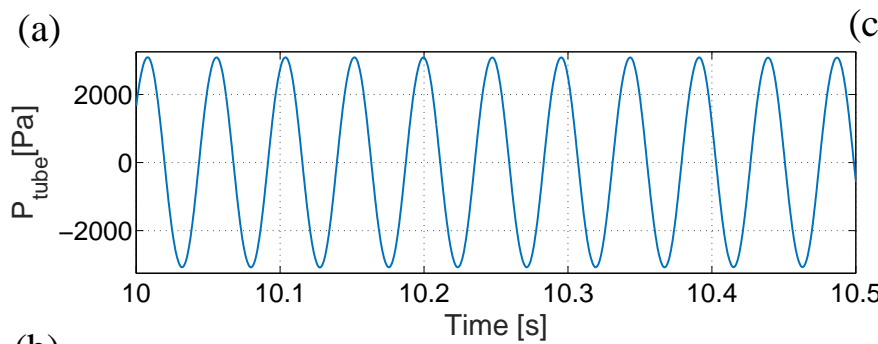

(c)

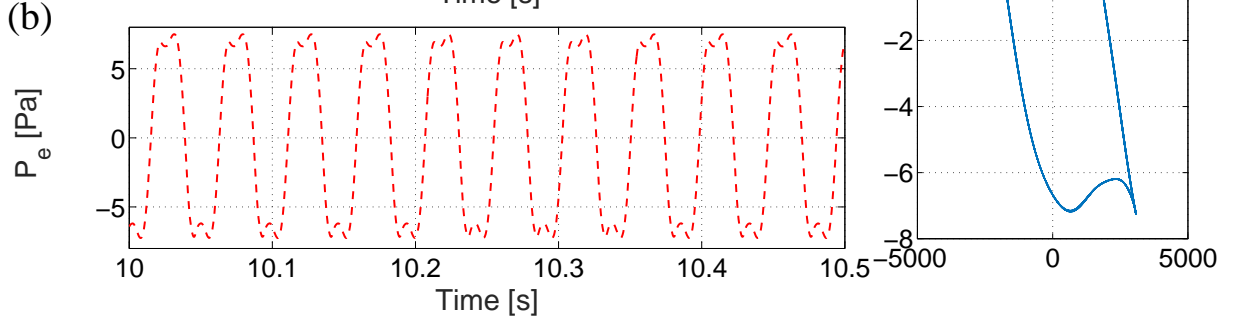

Figure 13: Idem as Fig. 11 with the excitation frequency $f_{\mathrm{e}}=83.5$ and excitation amplitude $E=2.24$.

The three highlighted families of response regimes are illustrated Figs. 11. 12 and 13 . Each figure includes three plots showing $p_{\text {tube }}(t)$ alone (top left), ${ }_{400} p_{\mathrm{e}}(t)$ alone (bottom left) and both of them in a $\left(p_{\text {tube }}(t), p_{\mathrm{e}}(t)\right)$ parametric plot named phase plane (right). Fig. 11 is typical of the responses to low excitation amplitudes, here $E=0.29$ and $f_{\mathrm{e}}=86.75 \mathrm{~Hz}$. As expected the time responses are sine curves corresponding to a classical elliptic simple closed curve in the phase plane. Figure 12 corresponds to a case where the EA-NES is efficient, $E=1.5$ and $f_{\mathrm{e}}=86.75 \mathrm{~Hz}$. The regime displays strongly modulated amplitudes in the time responses (SMR). As expected the curve in the phase plane is a torus. Fig. 13 shows a typical regime in the domain where EA-NES is not efficient, with $E=2.24$ and $f_{\mathrm{e}}=83.5 \mathrm{~Hz}$. The time responses are periodic. Harmonic effects are most visible on $p_{\mathrm{e}}$ and give a non-elliptic closed curve in the phase space. It is interesting to note that the phase shift between the two acoustic pressures $p_{\text {tube }}$ and $p_{\mathrm{e}}$ changes when the excitation amplitude increases. At low excitation amplitude, $p_{\text {tube }}$ and $p_{\mathrm{e}}$ shows a positive phase shift whereas the phase shift becomes negative for large excitation amplitude. This behavior is in accordance with the fact that the motion shifts from an in-phase nonlinear 
mode to an out-phase nonlinear mode when TET disappears [9].

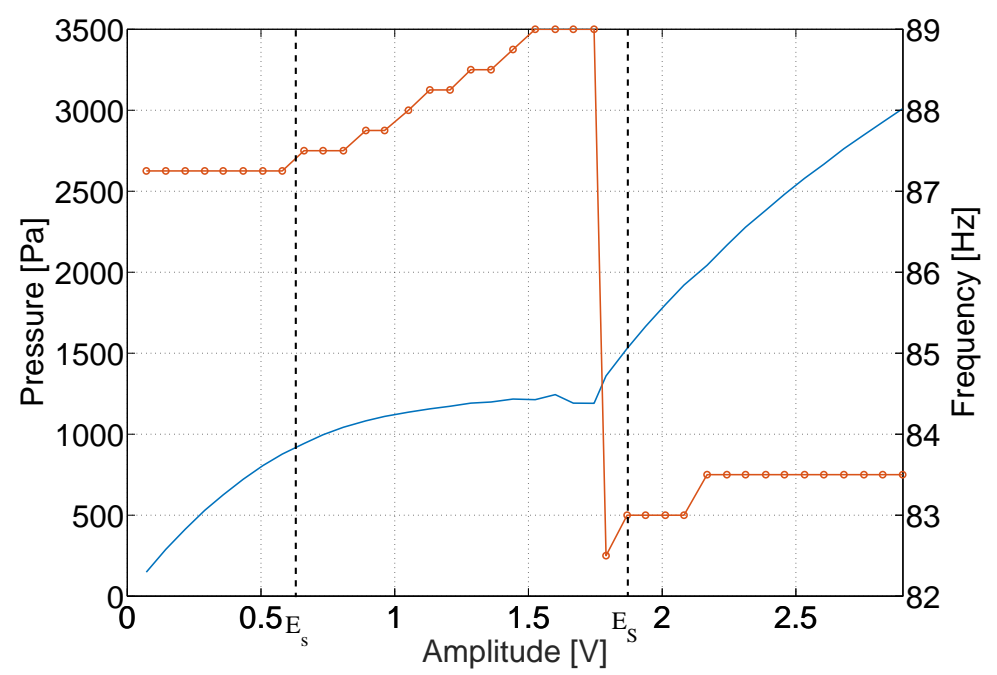

Figure 14: System with the EA-NES with $K=200$ coupled to the tube of $L_{1}$ length: Ridge line (blue line) of the acoustic pressure $p_{\text {tube }}$ and corresponding resonance frequencies (red with bullets line, $y$-axis on right side) versus the excitation amplitude.

The characteristic elements of the EA-NES behavior can be captured on the ridge line plot of the acoustic pressure $p_{\text {tube }}$. The ridge line is defined as the maxima of the RMS values of the acoustic pressure for the considered frequency range versus excitation amplitude. It is plotted as a blue continuous line in Fig. 14 (left axis). On the same plot the red bullet-line curve indicates the corresponding excitation frequencies (right axis) where the maxima of the RMS values of the acoustic pressure occurs. They are named hereafter resonance frequencies in the framework of modal analysis. The triggering TET threshold is defined as the excitation amplitude, here denoted $E_{\mathrm{s}}$, where the acoustic pressure increases more slowly than the underlying linear system (or equivalently as the excitation amplitude where the resonance frequency deviates from the resonance frequency associated to the tube of the underlying linear system). As shown in Fig. 7. $E_{\mathrm{s}} \simeq 0.6$ corresponding to $1000 \mathrm{~Pa}$ as the RMS value of $p_{\text {tube }}$. The threshold for the appearance of undesired periodic regimes, here denoted 
$E_{\mathrm{S}}$, is defined as the excitation amplitude where the variation of the acoustic pressure again increases corresponding to an abrupt change in the resonance frequency resulting in a smaller value. As shown in Fig. 7. $E_{\mathrm{S}} \simeq 1.8$. In the excitation amplitude band $\left[E_{\mathrm{S}}, E_{\mathrm{S}}\right]$, the acoustic pressure remains almost constant, $\left[E_{\mathrm{s}}, E_{\mathrm{S}}\right]$ defines the efficiency span of the EA-NES (i.e the excitation amplitude band where the EA-NES works well) and it corresponds to the domain where the SMR take place.

4.2. EA-NES with the tube of $L_{1}$ length: influence of $K$

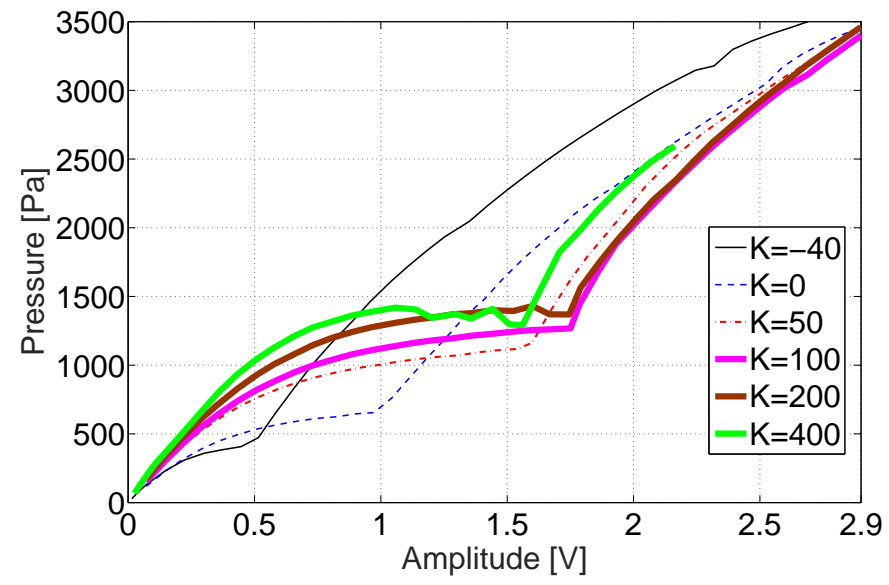

Figure 15: System with the EA-NES with several values of the gain $K$ coupled to the tube of $L_{1}$ length: Ridge line of the RMS values of $p_{\text {tube }}$ according to the level of excitation.

The same analysis was conducted for six values of the gain $K(=-40,0,50$, 100, 200 and 400). The analysis of the data, here only the associated ridge lines of the acoustic pressure $p_{\text {tube }}$ are reported (see Fig. 15), confirms that the values of $K$ have a great impact on the efficiency of the resulting EA-NES both on the TET thresholds and the resulting acoustic pressures. Starting from $K=-40$, we observe that increasing $K$ increases simultaneously $E_{\mathrm{s}}, E_{\mathrm{S}}-E_{\mathrm{s}}$ and the resulting acoustic pressure in the domain where the EA-NES works well. The 
EA-NES leads to a two-to-three decrease $(6$ to $10 \mathrm{~dB})$ in the acoustic pressure. The influence of the gain $K$ is similar to the influence of the linear stiffness of a classical NES 9 .

\subsection{EA-NES with the tube of $L_{2}$ length: influence of $K$}

The same analysis was also conducted with a tube of length $L_{2}$.
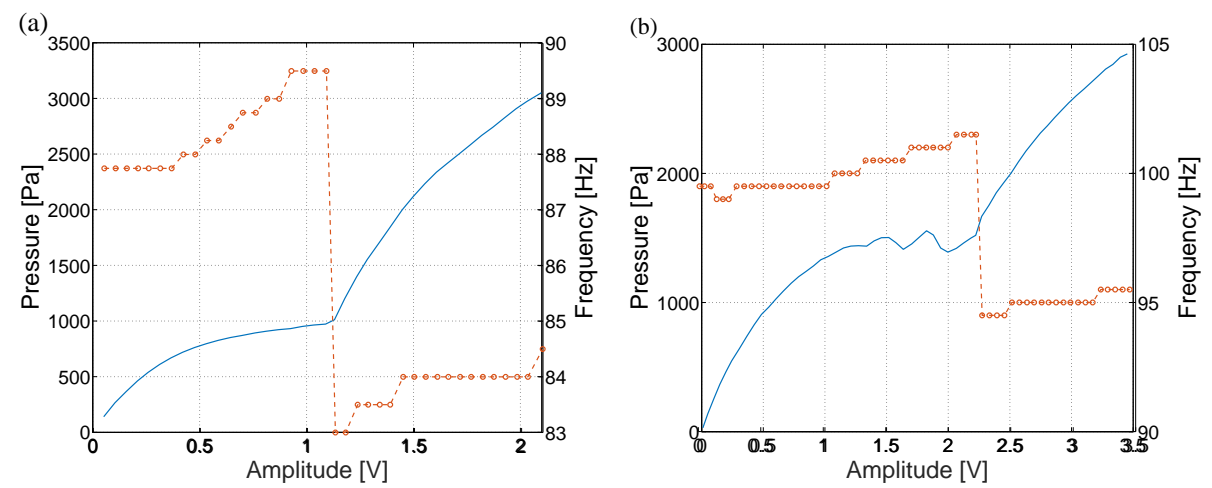

Figure 16: System with the EA-NES with the gain $K=50$ coupled to the tube of (a) $L_{1}$ and (b) $L_{2}$ length: Ridge line (blue line) of the acoustic pressure $p_{\text {tube }}$ and corresponding resonance frequencies (red with bullets line, $y$-axis on right side) versus the excitation amplitude.

A very important property of this EA-NES can be first highlighted: its ability to adapt and tune itself to the resonance frequency of different linear systems. To demonstrate experimentally this property we report in Fig. 16 the ridge lines of the acoustic pressure $p_{\text {tube }}$ measured on the system with the tube of $L_{1}$ length and on the system with the tube of $L_{2}$ length using the the EA-NES with the same gain value $K=50$. In both cases, we observe that EA-NES works well and the associated efficiency span $\left(\left[E_{\mathrm{s}}, E_{\mathrm{S}}\right]\right)$ are $[0.5,1.1]$ and $[1,2.2]$ respectively. However it appears that the EA-NES does not perform noise reduction with the same efficiency for the two tubes. Indeed, with the tube of $L_{1}$ length the acoustic pressure in the efficiency span is near $750 \mathrm{~Pa}$ whereas is about $1500 \mathrm{~Pa}$ for the tube of length $L_{2}$. This difference is due to the fact that the tube of $L_{2}$ length has a higher resonance frequency than the tube of $L_{1}$ length. In consequence, more energy has to be provided to the tube of length $L_{2}$ so that resonance capture occurs [17]. 


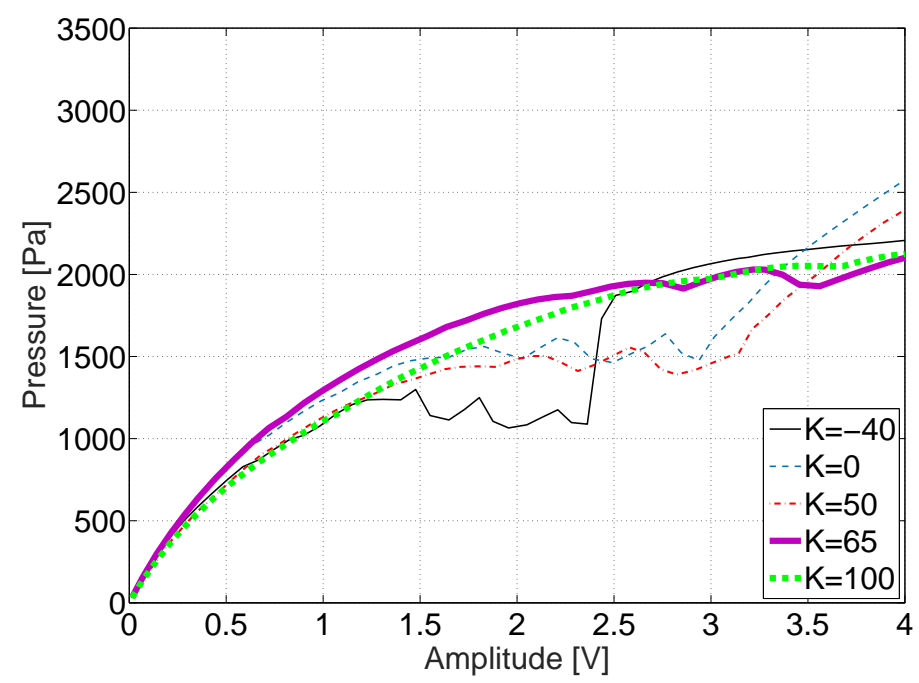

Figure 17: System with the EA-NES with several values of gain $K$ coupled to the tube of $L_{2}$ length: Ridge line of the RMS values of $p_{\text {tube }}$ according to the level of excitation.

Figure 17) shows the ridge lines of the acoustic pressure $p_{\text {tube }}$ obtained with five values of gain $K(=-40,0,50,65$ and 100). The results are similar to that observed with the tube of length $L_{1}$ for the configuration with the EA-NES with $K=-40,0$, and 50 where the thresholds $E_{s}$ and $E_{S}$ are visible. For the configuration with the EA-NES with $K>50$, only the threshold $E_{s}$ are reached. A complete excursion as in case of tube of $L_{1}$ length was not be possible due to a limitation of the acoustic source performance used in this setup.

Finally, the maximum power consumed by the control loudspeaker are displayed in Fig. 18 for several values of the gain $K$. For all cases, the power consumed by the loudspeaker increases with the excitation amplitude and slowly varies with the gain $K$ for positive values. The maximum power consumed by the loudspeaker is inferior to $1.5 \mathrm{~W}_{r m s}$. Thus the EA-NES requires a limited amount energy to work, which is a good asset for industrial applications. 


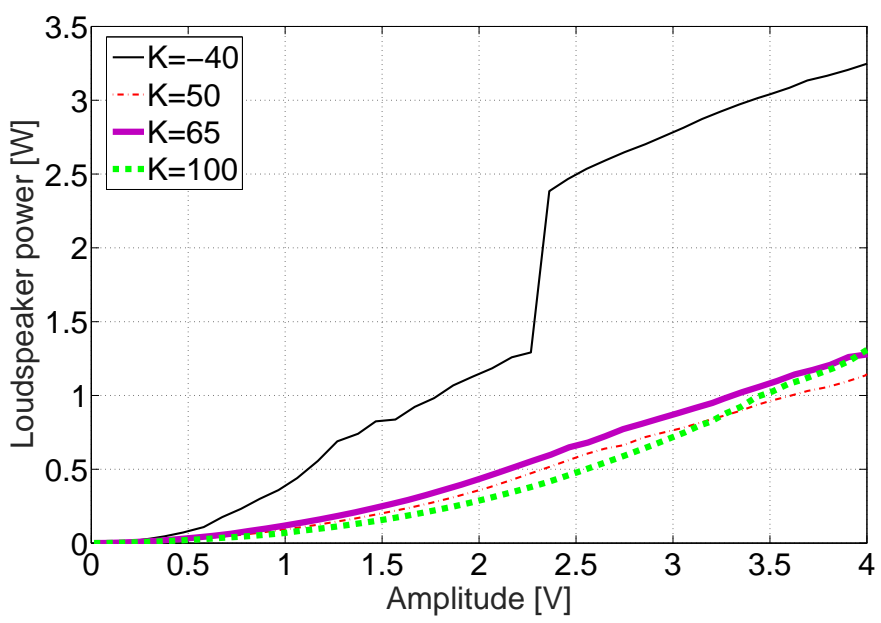

Figure 18: System with the EA-NES with several values of gain $K$ coupled to the tube of $L_{2}$ length: Maximum value of power consumed by the control loudspeaker depending on the level of excitation.

\section{Conclusion}

A new acoustic NES with a controlled acoustic load has been presented. The control of the linear stiffness of the membrane by the help of a feedback loop has been validated experimentally. Furthermore this NES has been tested coupled to two different tubes and has performed acoustic pumping with various efficiencies depending on the gain $K$. It appears that there is no optimum value of the gain $K$ because it depends on the excitation level. Indeed one can obtain either a low threshold but a short span when $f_{N E S}^{\prime}$ is close to $f_{T}^{\prime}$, or a high threshold with a large span when $f_{N E S}^{\prime}$ is distant from $f_{T}^{\prime}$. However, unlike the previous passive NES, the frequency of the EA-NES can be easily modified in real time. Future work is ongoing, focusing on using a gain $K$ variable. It would allow to optimize $K$ according to the excitation in order to get the strongest sound attenuation. We work also on the integration of the EA-NES in a cavity, with a narrow band noise excitation, in the framework of progress towards applications. 
This study opens the way for EA-NES research: the electrical drive of a control loudspeaker could be used to emulate unconventional hybrid activepassive NES. Its low consumption could render it more applicable than purely active devices, and raises the potential to harvest its energy consumption from the surrounding strong acoustic fields to damp.

\section{Acknowledgment}

The first author acknowledges DGA-France for the financial support. 


\section{References}

500

[1] A. Vakakis, O. Gendelman, L. Bergman, D. McFarland, G. Kerschen, Y. Lee, Nonlinear targeted energy transfer in mechanical and structural systems, Vol. 156 of Solid mechanics and its applications, Springer, 2008.

[2] A. Vakakis, O. Gendelman, Energy pumping in nonlinear mechanical oscillators: Part II - Resonance capture, Journal of Applied Mechanics 68 (2001) 42-48.

[3] Y. Starosvetsky, O. Gendelman, Dynamics of a strongly nonlinear vibration absorber coupled to a harmonically excited two-degree-of-freedom system, Journal of Sound and Vibration 312 (2008) 234-256.

[4] E. Gourdon, N. Alexander, C. Taylor, C.-H. Lamarque, S. Pernot, Nonlinear energy pumping under transient forcing with strongly nonlinear coupling: Theoretical and experimental results, Journal of Sound and Vibration 300 (2007) 522-551.

[5] G. Sigalov, O. Gendelman, M. AL-Shudeifat, L. Manevitch, A. Vakakis, L. Bergman, Resonance captures and targeted energy transfers in an inertially-coupled rotational nonlinear energy sink, Nonlinear Dyn 69 (2012) 1693-1704.

[6] E. Gourc, S. Seguy, G. Michon, A. Berlioz, B. Manne, Quenching chatter instability in turning process with a vibro-impact nonlinear energy sink, Journal of Sound and Vibration 355 (2015) 392-406.

[7] P.-O. Mattei, R. Ponçot, M. Pachebat, R. Côte, Nonlinear targeted energy transfer of two coupled cantilever beams coupled to a bistable light attachment, Journal of Sound and Vibration 373 (Supplement C) (2016) 29-51. doi:https://doi.org/10.1016/j.jsv.2016.03.008.

[8] B. Cochelin, P. Herzog, P.-O. Mattei, Experimental evidence of energy pumping in acoustics, C. R. Mécanique 334 (11) (2006) 639-644. 
[9] R. Bellet, B. Cochelin, P. Herzog, P.-O. Mattei, Experimental study of targeted energy transfer from an acoustic system to a nonlinear membrane absorber, Journal of Sound and Vibration 329 (2010) 2768-2791.

[10] R. Mariani, S. Bellizzi, B. Cochelin, P. Herzog, P.-O. Mattei, Toward an adjustable nonlinear low frequency acoustic absorber, Journal of Sound and Vibration 330 (2011) 5245-5258.

[11] R. Cote, M. Pachebat, S. Bellizzi, Experimental evidence of simultaneous multi-resonance noise reduction using an absorber with essential nonlinearity under two excitation frequencies, Journal of Sound and Vibration 333 (2014) 5057-5076.

[12] J. Shao, B. Cochelin, Theoretical and numerical study of targeted energy transfer inside an acoustic cavity by a non-linear membrane absorber, International Journal of Non-linear Mechanics 64 (2014) 85-92.

[13] R. Boulandet, H. Lissek, Optimization of electroacoustic absorbers by means of designed experiments, Applied Acoustics 71 (2010) 830-842.

[14] H. Lissek, R. Boulandet, R. Fleury, Electroacoustic absorbers : Bridging the gap between shunt loudspeaker and active sound absorption, J. Acoust. Soc. Am 129 (2011) 2968-2978.

[15] R. Boulandet, H. Lissek, Toward broad band electroacoustic resonators through optimized feedback control strategies, Journal of Sound and Vibration 333 (2014) 4810-4825.

[16] O. Lacour, M. Galland, D. Thenail, Preliminary experiments on noise reduction in cavities using active impedance changes, Journal of Sound and Vibration 230 (1) (2000) 69-99.

${ }_{550}$ [17] R. Bellet, Vers une nouvelle technique de controle passif du bruit : Absorbeur dynamique non lineaire et pompage energetique, $\mathrm{Ph} . \mathrm{D}$. thesis, Universite de Provence (2010). 
[18] R. Bortoni, S. Filho, N. Sidnei, H. Silva, Effects of acoustic damping on current-driven loudspeakers, in: 122nd Convention of the Audio Engineering, no. Paper number: 7073, Vienna (Austria), 2007. 